\title{
LA EVOLUCIÓN DE LA POLÍTICA TURÍSTICA EN ARGENTINA. UN ANÁLISIS DE SUS FINALIDADES ${ }^{1}$ \\ The Evolution of Tourism Policy in Argentina. An Analysis of its Purposes
}

\author{
Erica Schenkel $^{2}$ \\ Recibido: mayo, 2018 // Aceptado: junio, 2018
}

\section{RESUMEN}

Desde el análisis de políticas públicas, el artículo aborda la evolución de la política turística argentina entendiendo tal política como parte de un proceso histórico. La metodología que acompaña esto consiste en el análisis documental de normativas, leyes, decretos y documentos enfocados en la evolución de la política turística argentina. La misma responde a un contexto social, económico y político particular, estructurado sobre la base de cánones internacionales vigentes, que permiten identificar periodos diferenciados, que no se pueden explicar a partir de una única variable. Las acciones públicas en el área llevan consigo las ideas y necesidades del gobierno y las relaciones de este con los demás actores gubernamentales y privados, que dinámicamente ocupan una posición predominante en la arena turística. El estudio identifica: una fase inicial, previa al surgimiento histórico del turismo como asunto público, a partir de una fuerte participación de actores privados; una fase de expansión, asociada a la consolidación del Estado de Bienestar argentino y la generalización del derecho a las vacaciones pagadas; y finalmente, una fase de reconversión, vinculada a las ideas de desregulación y privatización que priman desde finales del siglo XX, consolidando las finalidades económicas asociadas al impulso de la actividad.

Palabras clave: Análisis de política pública; Política turística; Evolución; Argentina

\section{ABSTRACT}

From the analysis of public policies, the article discusses the evolution of Argentina's tourism policy understanding such policy as part of a historical process. The methodology that accompanies this consists in the documentary analysis of regulations, laws, decrees and documents focused on the evolution of Argentina's tourism policy. This responds to a particular social, economic and political context, structured on the basis of current international canons, which allow differentiated periods to be identified, which

${ }^{1}$ El artículo fue presentando como ponencia en el X Congreso de la Sociedad de Investigadores en Turismo de Chile. El mismo describe la evolución de la política turística argentina en una versión resumida. Para ampliar dicha información consultar Schenkel (2017).

${ }^{2}$ Doctora en Ciencias Sociales. Universidad Nacional del Sur y CONICET. Líneas de Investigación: Turismo; Política turística; Turismo social. Dirección: La Falda 128, (8000) Bahía Blanca, Argentina. Teléfono: +54 291 4198075. E-mail: +54 2914198075. 
cannot be explained by a single variable. Public actions in the area carry with them the ideas and needs of the government and its relations with other governmental and private actors, which dynamically occupy a predominant position in the tourism arena. The study identifies: an initial phase, prior to the historical emergence of tourism as a public issue, based on the strong participation of private actors; an expansion phase, associated with the consolidation of the Argentine welfare state and the generalization of the right to paid holidays and, finally; a phase of reconversion, linked to the ideas of deregulation and privatization that prevail since the end of the 20th century, consolidating the economic purposes associated with the promotion of the activity.

Key words: Policy Analysis; Tourism Policy; Evolution; Argentina

\section{INTRODUCCIÓN}

La escasez de análisis politológicos en torno al turismo se explica por la primacía de la perspectiva económica que concibe al fenómeno como una simple actividad de consumo -ligada al sector privado-, prevaleciendo en su análisis la aplicación de teorías de gestión empresarial, que reducen la política turística a planes de marketing. Sin embargo, el turismo conforma una praxis social con una singular estructura y proceso político, que involucra diferentes elementos de naturaleza pública, asociados directa e indirectamente a aspectos teóricos y prácticos de la política y de la administración gubernamental (Matthews \& Richter, 1991).

La relevancia en términos prácticos de conocer los resultados de ciertas políticas turísticas ha llevado a reconocer de manera cada vez más enfática la necesidad de emprender estudios vinculados a su análisis político (Jafari, 2002; Scott, 2011). El estudio de políticas públicas presenta una orientación fundamentalmente práctica, que es articulada en tres dimensiones: una aproximación empírica referida a las causas y los efectos de la política en torno a hechos; una evaluativa, tendiente a indagar el valor de la iniciativa; y una normativa, que refiere a las futuras acciones que puedan llegar a optimizar la acción pública (Pérez Sánchez, 2010).

El análisis que se presenta en las siguientes páginas, parte de los abordajes amplios de evaluación, que pretenden explicar los resultados de la acción pública y sus efectos en la sociedad considerando: la complejidad del análisis, el carácter intrínsecamente histórico de los procesos y la necesidad de abordajes holísticos, que se extiendan a todo el ciclo de la política, a los procedimientos y metodologías utilizadas -incluyendo 
técnicas cuantitativas y cualitativas de indagación- (Oszlak y O’Donnell, 1995; Franco \& Lanzaro, 2006; Pérez Sánchez, 2010).

Desde esta perspectiva, la investigación aborda la evolución de la política turística argentina como parte de un proceso social. Aquello que se inició como una práctica privada, exclusiva de círculos de elite, fue adquiriendo significancia pública con su progresiva democratización, para pasar a ocupar una posición preponderante entre las políticas nacionales décadas después. El análisis identifica: funciones territoriales de la política turística argentina (1880-1930), relacionadas con la consolidación de la soberanía nacional ante un Estado en incipiente desarrollo; funciones sociales (1930-1976), vinculadas a la democratización de las prácticas turística a partir de una estructura social ascendente; y funciones económicas (desde 1976), asociadas al turismo como factor de crecimiento y de internacionalización, con un predominio del turismo receptivo. Si bien estas funciones públicas se suelen presentar concurrentemente, presentando matices y límites difusos, una prevalece en cada periodo.

\section{ASPECTOS METODOLÓGICOS}

Partiendo de una perspectiva integral, la investigación pretende explicar la evolución de la política turística argentina, identificando las acciones de los gobiernos en el área y las relaciones de estos con los demás actores políticos y económicos, en el marco de los factores extrínsecos que le confieren sentido y contenido político. Para alcanzar el objetivo mencionado, se plantean los siguientes objetivos específicos:

- Identificar los intereses públicos asociados a la política turística argentina en el devenir histórico.

- $\quad$ Reconocer los principales actores participantes en dicho proceso.

- $\quad$ Periodizar la evolución de esta política argentina dando cuenta de las diferentes etapas que circunscriben su desarrollo.

El estudio parte del análisis de políticas como enfoque teóricometodológico. Gelambí Torrel (2006, p. 198) lo define como una metodología propia de la ciencia política que permite "aproximarse a la realidad de forma práctica y objetiva mediante el estudio del diseño de sus productos políticos”. Las políticas públicas se consideran “acordes de un 
proceso social” (Oszlak \& O’Donnell, 1995, p. 110), un conjunto de respuestas que analizadas en un contexto y momento determinado, permiten inferir la posición "predominante” del Estado en torno a una cuestión. Desde esta perspectiva, el abordaje de las políticas estatales debe contribuir a desagregar y poner en movimiento a un Estado configurado en un proceso social en el que se relaciona con otras fuerzas sociales (Gelambí Torrel, 2006).

Entre las técnicas de investigación se destaca el análisis documental. El mismo utiliza distintos tipos de fuentes: normativas, constituciones, leyes, decretos y resoluciones; estadísticas, Registros del Municipio de General Pueyrredón y Encuesta de Turismo Internacional; manifestaciones oficiales: Memorias Detalladas del estado de la Nación, Informes Anuales de Gestión, Planes de Turismo, escritos y prensa pública; documentos de especialistas en el área, Organización Mundial del Turismo (OMT), otros organismos gubernamentales y no gubernamentales internacionales y especialistas académicos-investigadores.

A partir de este corpus de investigación se realiza un análisis longitudinal de la política turística argentina, que permite explicar su origen y consolidación en la agenda de gobierno e identificar las diferentes etapas que circunscriben su evolución. La periodización sigue lapsos históricos a partir de modelos de acumulación (Torrado, 2010). Trazando líneas de continuidad y ruptura entre diferentes momentos de la historia contemporánea, el análisis concluye con un esquema síntesis que caracteriza cada una de las etapas evolutivas como parte de un proceso social más amplio.

\section{FINALIDADES PÚBLICAS ASOCIADAS A LA POLÍTICA TURÍSTICA ARGENTINA}

Los diversos intereses asociados al turismo originaron que el Estado argentino comenzara tempranamente a articular decisiones vinculadas a la cuestión turística, constituyendo uno de los primeros países de la región en entender al turismo como una cuestión gubernamental, ya en las primeras décadas del siglo XX. Su evolución responde a un contexto social, económico y político particular, que permite identificar periodos diferenciados, que no se pueden explicar a partir de una única variable (Schenkel y Almeida, 2015). Aquello que se inició como una práctica privada, exclusiva de círculos de elite, fue adquiriendo significancia 
pública con su progresiva democratización, para pasar a ocupar una posición preponderante entre las políticas nacionales medio siglo después (Figura 1).

Se identifican tres fases en la evolución de la política turística argentina: (i) fase inicial (1880-1930), que circunscribe un periodo de descubrimiento del turismo, previo al surgimiento histórico de la cuestión como problema público, con finalidades territoriales asociadas al desarrollo de la actividad y la participación mayormente de actores privados; (ii) fase de expansión (1930-1976), que refiere al proceso de democratización de las prácticas turísticas, que concierne a un momento de fuerte intervención estatal, en la cual el turismo adquiere finalidades sociales, a partir de la proclamación del derecho a las vacaciones pagadas; y ${ }^{\text {(iii) }}$ fase de reconversión (desde 1976), cuando la cuestión turística se adapta a las ideas de desregulación y privatización que priman desde finales del siglo XX, consolidándose las funciones económicas asociadas al impulso de la actividad y ocupando el turismo receptivo un lugar preponderante. Si bien estas funciones públicas se suelen presentar concurrentemente, presentando matices y límites difusos, una prevalece en cada periodo.

Figura 1. Consolidación de la política turística argentina en el devenir histórico

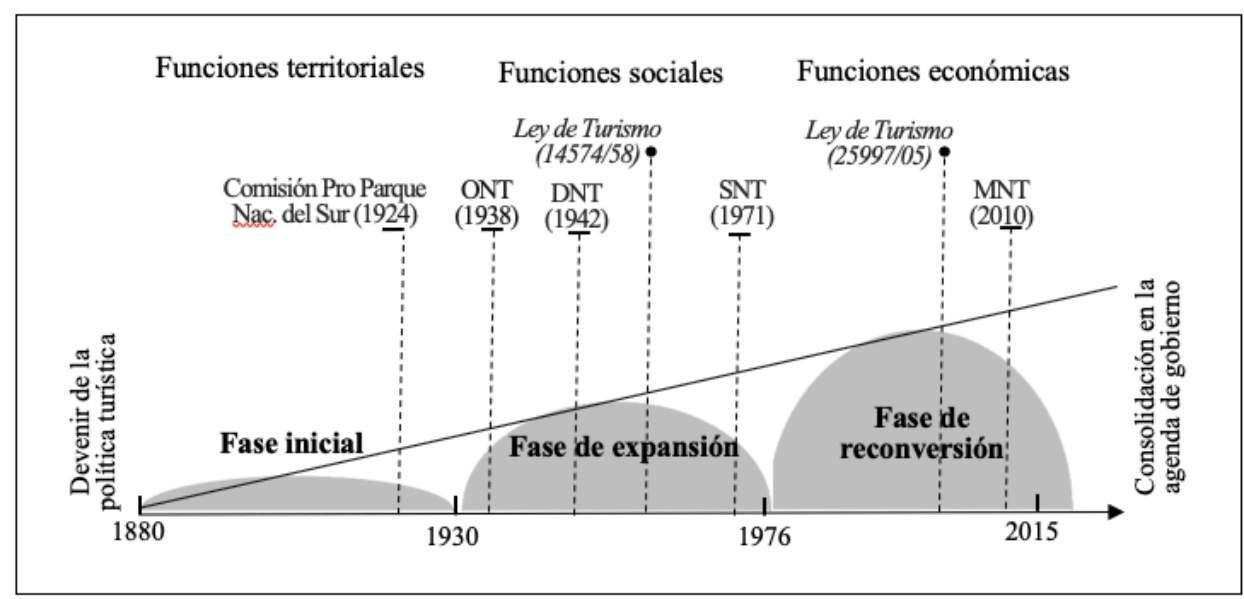

Fuente: Elaboración propia. 
IV. FASE INICIAL (1880-1930). FINALIDADES TERRITORIALES ASOCIADAS A LA POLÍTICA TURÍSTICA

La Fase Inicial circunscribe un periodo de descubrimiento de la actividad turística; anterior a la problematización del turismo como asunto público. A fines del siglo XIX, Argentina se incorporó al mercado mundial como exportador de materias primas agropecuarias e importador de manufacturas (Ferrer, 2010; Rapoport, 2010). Ante un Estado con extensos territorios despoblados, se comenzó a desarrollar un interés gubernamental por la actividad turística desde una dimensión territorial, como aseguradora de la soberanía nacional. Preocupada por asociar esta consolidación del Estado al "progreso" y a la "modernidad” europea, la elite gobernante inició el desarrollo de infraestructura básica, que sirvió de sostén a la expansión del turismo en el siguiente periodo (Schenkel, 2017).

El impulso de áreas naturales en sitios de frontera se llevaba a cabo junto con la localización de grupos poblacionales, en algunos casos incluso con la instalación de guarniciones militares, con el objetivo de ocupar extensos territorios despoblados. El turismo se incorporó a la política de protección ambiental mediante la aprobación del Parque Nacional del Sud (1922), primer Parque Nacional en Latinoamérica y tercero a nivel mundial, y la creación del primer órgano público con competencias asociadas, la Comisión Pro Parque Nacional del Sud (1924). En esta línea, se promovió la ampliación de las líneas férreas a lo largo del territorio nacional y se inauguró el puerto de Buenos Aires (1889).

Al calor del modelo agroexportador se consolidó una aristocracia terrateniente vinculada a la elite gobernante, que comenzó a realizar viajes con necesidades de recreación y salud: las largas estadías en Europa se intercalaban con el veraneo en segundas residencias a orillas del Río de La Plata y la práctica de baños termales (Schlüter, 2003). Argentina se convirtió en uno de los primeros países americanos en desarrollar centros turísticos, junto a Estados Unidos (Atlantic City), Chile (Viña del Mar) y, posteriormente, Uruguay (Pocitos, Piriápolis y Punta del Este) y Brasil (Río de Janeiro), que contaban con fuerte presencia de inmigrantes y así con la costumbre de vacacionar (Campodónico y de Cunha, 2012; Schenkel, 2017). Como enclaves de ocio aristocráticos surgió Mar del Plata y, posteriormente, otro conjunto de destinos: atlánticos, de la costa bonaerense, y serranos, de las provincias de Córdoba y Mendoza. Era un turismo de elite, socialmente minoritario y selecto, con un crecimiento 
limitado y un impacto puntual desde el punto de vista económico (Figura 2).

Figura 2. Evolución de los turistas alojados en establecimientos turísticos de Mar del Plata ${ }^{3}$

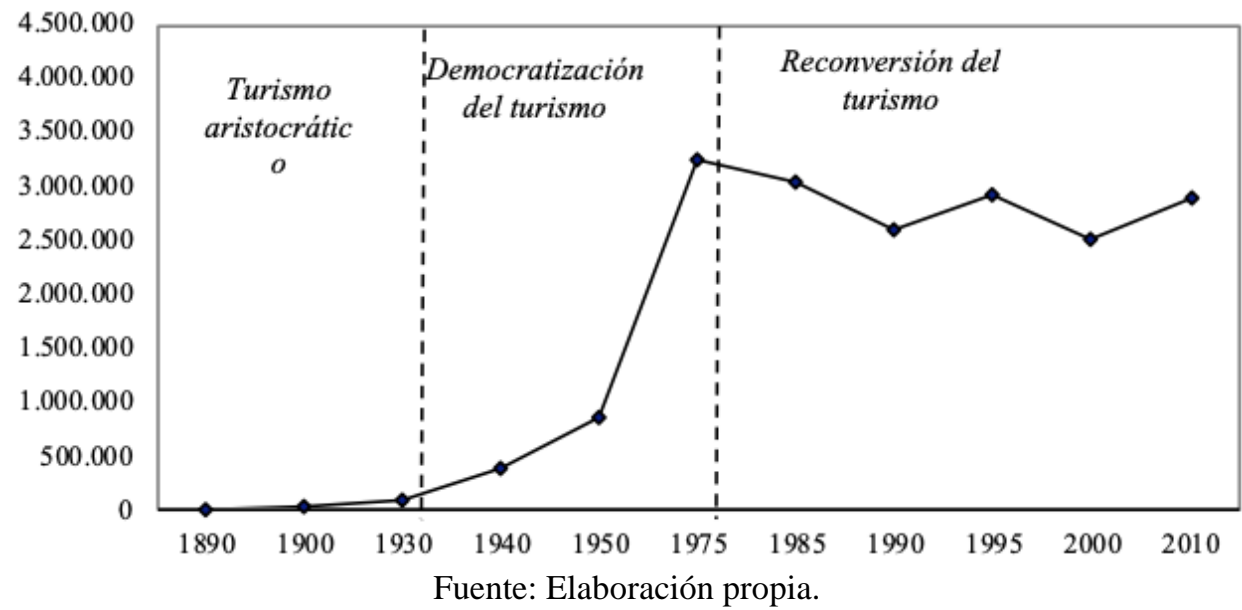

En este incipiente desarrollo de la actividad fue clave la participación de los actores privados, con fuerte vínculo con la aristocracia local y sus intereses de clase: por un lado, las empresas británicas a cargo del transporte férreo, que ampliaron la conectividad entre los principales centros del país, emprendieron la construcción de infraestructura e impulsaron la publicidad turística en distintos medios de difusión; y por otro, el Touring Club Argentino (TCA) y el Automóvil Club Argentino (ACA), que cumplieron una función decisiva con la difusión del automóvil, proveyendo mapas ruteros, estaciones de servicios con albergues e información específica. Estas últimas organizaciones facilitaron la consolidación de numerosos enclaves balnearios en la costa próxima de Buenos Aires, así como la difusión de segundas residencias (Pastoriza, 2011).

\footnotetext{
${ }^{3}$ Mar del Plata es el clásico destino turístico de sol y playa, ubicado en la Costa Atlántica Bonaerense. Desde sus inicios constituye el principal destino del turismo nacional convirtiéndose en referencia al momento de analizar el comportamiento turístico de los argentinos, ante la falta de estadísticas nacionales.
} 
V. FASE DE EXPANSIÓN (1930-1976). EL DERECHO A LAS VACACIONES

La Fase de Expansión (1930-1976) refiere al proceso de democratización de las prácticas turísticas, que permitió un crecimiento exponencial de la actividad, a partir de una estructura social ascendente. La crisis internacional de 1930 motiva el impulso de un nuevo modelo de acumulación basado en una industria de sustitución de importaciones, que sustenta las bases para la creación del Estado de Bienestar argentino (Ferrer, 2010). El crecimiento de las actividades no agropecuarias, origina un aumento de las tasas de empleo, causando un proceso de movilidad ascendente, que conforma la estructura social moderna. El ascenso de las clases medias y sectores populares permitió la democratización de bienes y servicios antes exclusivos de la "oligarquía”, entre los que se incluyen las prácticas turísticas.

En esta “democratización del turismo” (Pastoriza, 2011) fue clave el accionar del colectivo obrero, forzando mejoras laborales asociadas al descanso y al ocio, articulando con los programas estatales y, en muchos casos, construyendo infraestructura turística propia, a partir de sus sindicatos. A los primeros centros turísticos, se incorporan nuevos destinos de la Costa Atlántica, como Villa Gesell, San Clemente del Tuyú, San Bernardo, Pinamar y Santa Clara del Mar, y otros de las sierras cordobesas, destacándose La Falda, Los Cocos, Cosquín, Alta Gracia, Villa Carlos Paz, Capilla del Monte y Mina Clavero. Es la etapa del paso de un modelo de ocio minoritario a otro democrático, asociado a la ampliación de la distribución del ingreso (Mantero, 1997). Estos viajes de veraneo se caracterizan por largas estadías, una fuerte estacionalidad estival y la utilización del ferrocarril como medio de transporte, que luego compite con el automóvil particular y el ómnibus (Schlüter, 2003). La hotelería lujosa es desplazada por formas de alojamiento más modestas, como los hoteles estatales y sindicales, las viviendas particulares de uso turístico y el camping.

La progresiva democratización de las prácticas de ocio ocasiona que la cuestión vaya adquiriendo significancia pública. La Oficina Nacional de Turismo (1938) fue el primer órgano público con competencias específicas en el país y uno de los primeros en constituirse en América Latina, junto a la Comisión Mixta Pro-Turismo de México (1922) y la Comisión Nacional de Turismo de Uruguay (1935); 
posteriormente, se crea la Oficina de Turismo de Venezuela (1947), la Dirección de Turismo en Chile (1960) y la Empresa Brasileira de Turismo (1966).

En 1941 se establece la Comisión Nacional de Turismo, bajo la órbita del Ministerio de Agricultura, para poco después convertirse en Dirección Nacional de Turismo (1942). A partir de entonces, se instauran los primeros organismos provinciales: Tucumán (1936), Mendoza (1936), Córdoba (1938), La Rioja (1939), Santiago del Estero (1940), San Luis (1946), Buenos Aires (1948), Entre Ríos (1950), Catamarca (1953), San Juan (1955), Neuquén (1961), Río Negro (1963), Chubut (1964) y La Pampa (1969).

La llegada de Juan Domingo Perón al poder en 1943 provocó un cambio abrupto en los diferentes ámbitos nacionales, a partir de un ambicioso proceso de redistribución del ingreso nacional, se promueve el ascenso social de los sectores populares -ampliando el concepto de ciudadanía más allá de los derechos políticos- (James, 2010). El gobierno jerarquizó el área y trató al turismo como una política pública específica por primera vez en el marco nacional. Este momento de institucionalización se pretendió cristalizar con la sanción de la primera Ley Nacional de Turismo, norma que quedó trunca con el golpe de Estado de 1955.

La histórica Dirección de Parques Nacionales, a la cual el peronismo le asignaba vínculos con el “interés oligárquico” (Presidencia de la Nación, 1953), pasa a denominarse Administración de Parques Nacionales y Turismo (decreto 9504/45), un ente autárquico dependiente del Ministerio de Obras Públicas. El nuevo organismo incluyó competencias relacionadas con el turismo y los hoteles bajo su jurisdicción se incorporan a las listas de hospedajes estatales, como sucede con los transferidos con la estatización de los ferrocarriles y aquellos cedidos por las provincias.

En este periodo el turismo adquirió una destacada función social en la agenda gubernamental, buscando contribuir a la reivindicación de los sectores marginados con la generalización del derecho a las vacaciones pagadas (decreto 1740/45). El turismo social se inserta en la agenda del gobierno -asignando recursos públicos específicos- con diferentes iniciativas, como la construcción de complejos estatales para aquellos colectivos beneficiados con las vacaciones pagadas. Las acciones 
consolidan un proceso de ampliación social del ocio que se inicia en los años treinta con el avance de las clases medias, destacando al país en el marco regional, por los altos niveles de participación turística. Esta situación se manifiesta en el fuerte crecimiento del principal destino nacional que es Mar del Plata (Figura 2).

El golpe de Estado de 1955, origina un extenso periodo de proscripción del peronismo, en el que se alternan gobiernos militares y civiles, basados en el "régimen semi-democrático" (Cavarozzi, 1983). Los nuevos gobiernos ponen en marcha un modelo económico desarrollista centralizado en el capital externo, que introduce la actividad turística dentro del pensamiento económico como alternativa de carácter técnico para el desarrollo nacional (Capanegra, 2011). Paralelamente a esta visión económica del turismo que comienza a aparecer en la arena pública, continua el proceso de ampliación social del ocio, impulsado por la estructura social ascendente que caracteriza al país hasta mediados de los ’70 (Figura 2).

En este marco se consolida la planificación en el sector: la Dirección Nacional de Turismo (DNT) pasa a ser una entidad descentralizada con fondos propios (1957) y se promulga la primera Ley Nacional de Turismo (14574/58) y su decreto reglamentario (9468/61). Del mismo modo, se sancionan las leyes nacionales de hotelería (18828/70), de agencias de viaje (18829/70) y de fomento turístico (18674/70). Posteriormente, la DNT asciende a Secretaría de Turismo (1971), dependiendo directamente de Presidencia.

\section{INTERNACIONALIZACIÓN DEL TURISMO COMO ACTIVIDAD ECONÓMICA (DESDE 1976)}

En la Fase de Reformulación, la cuestión turística se adapta a las ideas de desregulación y privatización que primaron desde finales del siglo XX. En el marco de la crisis del petróleo, el golpe de Estado de 1976 impulsa un modelo de producción de corte liberal, profundizado por los sucesivos gobiernos democráticos, que pone fin al Estado de Bienestar argentino (Ferrer, 2010; Rapoport, 2010). La economía sufre fuertes oscilaciones que repercuten negativamente en la estructura productiva. La base social consolidada en los años 40, se desintegra, el modelo de acumulación y producción aperturista socava las estructuras laborales formales, aumentando la pobreza y concentrando el ingreso (FIDE, 2004). 
En este contexto se produce una precarización de los derechos sociales, incluyendo aquellos ligados al acceso a la recreación y al turismo, que inicia una etapa de repliegue y redefinición de la actividad, en cuanto al turismo doméstico -luego de cincuenta años de expansión- (Figura 2). Paralelamente al declive de los destinos populares, surgen nuevos enclaves para elites nacionales en la Costa Atlántica Bonaerense y otros destinos nacionales, dando cuenta del proceso de fragmentación social que identifica al periodo. En una misma línea, se imponen productos turísticos alternativos, como el esquí, la fauna marina, la gastronomía, los carnavales, los trenes turísticos, los cruceros, los congresos y convenciones, que responden a las nuevas motivaciones.

Los arribos internacionales en tanto, crecen de manera sostenida, prácticamente se cuadriplican entre 1985 y 2011, como así también las salidas de argentinos al exterior (ETI, 1985-2013). El turismo receptivo, se constituye en una actividad complementaria de crecimiento económico, tendiente a atraer las necesarias divisas internacionales, ante la deficitaria balanza de pagos y la abultada deuda externa.

Asociada a la consolidación del sector turístico, la política turística se constituye en un factor de crecimiento, predominando su función económica. Los gobiernos ponen en marcha actuaciones similares a las realizadas por otros países, encontrando las referencias necesarias en las recomendaciones de los organismos internacionales, como el Fondo Monetario Internacional (FMI), la Organización de Estados Americanos (OEA) y la Organización Mundial de Turismo (OMT), que aconsejan una mayor liberalización del sector y una menor intervención estatal, en el marco de la financiación de proyectos turísticos (Capanegra, 2011). Este giro de la política turística se extiende en todos los países de la región, suponiendo abandonar un patrón turístico y económico más endógeno por otro que implica mayor dependencia externa.

En Argentina se aprueba el estatuto de la OMT con la ley 19644/72, pasando a integrar el organismo en 1975, con el objetivo de instituir al país como destino internacional. En esta línea, se implementan Planes de Marketing Estratégicos (1991-1993, 1994-1996 y 1997-1999), a partir de asistencia técnica y financiera de la Unión Europea; y se publica el primer documento de planificación pública del turismo: "Bases para un Plan Federal de Turismo” (1984). Paralelamente, se activa la participación privada en el sector, a través de mesas de colaboración y concertación 
(Reuniones Nacionales de Turismo), surge la Asociación de Hoteles de la República Argentina - AHT (1977) y la Cámara Argentina de Tiempos Compartidos - CATC (1984). Los procesos de privatización de la década del `90 incluyen sectores vinculados a la actividad turística, como los de aeronavegación y ferrocarriles y la concesión de la red vial, al mismo tiempo que se produce el arribo al país de las principales cadenas hoteleras y turoperadores.

El periodo de poscrisis del año 2001, circunscribe un proceso definido dentro de esta última fase, a partir de un reposicionamiento del Estado Nacional como actor clave en la planificación, coordinación y acción en materia turística. El turismo pasa a formar parte de las doce exclusivas políticas de Estado consolidando su importancia en la agenda gubernamental (Figura 1). La política turística se considera un instrumento central del Estado Nacional para alcanzar la reactivación económica:

La delicada situación coyuntural que afrontamos exige acelerar la marcha y replantear profundamente los fundamentos de la gestión. El Turismo es un poderoso inductor del desarrollo económico, social y cultural. En las actuales circunstancias del país esta capacidad para generar riqueza y mejorar la calidad de vida de los vastos sectores de la población es de primordial trascendencia. La Argentina tiene capacidad para acoger una cantidad mucho mayor de viajeros que los que hoy nos visitan. (Memoria del estado de la Nación del 2003:221)

La sanción de la nueva Ley Nacional de Turismo (25997/05) y su decreto reglamentario (decreto 1297/06), constituyen el hecho institucional de mayor relevancia en cuarenta años, al reemplazar la antigua ley 14574 de 1958. Con la nueva norma, el turismo deja de ser institucionalizado como actividad de ocio y recreación (ley 14574) para ser proclamado como una verdadera actividad económica. Declara de “interés nacional” al turismo como "actividad socioeconómica, estratégica y esencial”, siendo esta "prioritaria dentro de las políticas de Estado" (ley 25997, art. 1, 3). El turismo receptivo se distingue como una "actividad de exportación” no tradicional y el sector privado como una "aliado estratégico”, impulsando herramientas económicas similares a las que se otorgan a la actividad industrial, entre las que se destaca la creación de un instituto específico de promoción: el INPROTUR (Instituto Nacional de Promoción Turística).

En este marco, el gobierno impulsa el Plan Federal Estratégico de Turismo (PFETS), de carácter descentralizado y federal, tendiente a 
consensuar un modelo turístico a largo plazo entre los diferentes actores involucrados en el sector. El desarrollo del PFETS (SECTUR, 2005) en principio se planifica con horizonte al año 2016, luego, con su actualización (PFETS, 2011), al año 2020. A partir de talleres regionales, en los cuales participan actores públicos de los diferentes niveles de gobierno, privados y académicos, se definen los ejes rectores en materia turística nacional.

La histórica Secretaría de Turismo de la Nación crece en autoridad y competencias. En primera instancia se adscribe al Ministerio de Producción, que reúne aquellas áreas con "fuerte sesgo productivo": agricultura, ganadería y pesca, industria, turismo y comercio exterior (decreto 2025/08); posteriormente al Ministerio de Industria (decretos 1365/09 y 1366/09), luego denominado Ministerio de Industria y Turismo (decreto 1458/09), que integra turismo con las áreas de industria y comercio exterior; finalmente, a partir de la trascendencia económica que se le atribuye a la actividad, se convierte en un órgano ministerial autónomo por primera vez en la historia argentina, como Ministerio Nacional de Turismo (decretos 919/10 y 921/10), constituyendo el decimocuarto ministerio del Estado Nacional (Figura 1).

\section{CONCLUSIONES PRINCIPALES}

El análisis refleja la importancia que ha ocupado la cuestión turística en la agenda pública argentina. Las funciones asumidas por el Estado en el área permiten identificar fases diferenciadas que, ajenas a las alternancias de gobiernos, responden a una secuencia de alianzas y coaliciones conformadas con diferentes grupos de interés. Estas estrategias público-privadas, que dinámicamente ocuparon una posición preponderante en los procesos de toma de decisión, determinaron los fundamentos de la inclusión del turismo a la agenda de gobierno, condicionando la política turística a implementar. Aquello que se había iniciado como una cuestión exclusiva de una aristocracia terrateniente que exhibía fuertes vínculos con la elite gobernante y los actores privados que sustentaron los inicios de la actividad-, trascendió como problemática social décadas después, a partir de las conquistas laborales de los sectores obreros, que incluyeron entre sus reivindicaciones a aquellas necesidades asociadas al descanso y al ocio, para reconvertirse, finalmente, en una 
problemática asociada al crecimiento económico y a la internacionalización, a partir de la preponderancia que adquiere un sector turístico ya consolidado, que comienza a pujar por cuestiones de rentabilidad.

La Fase Inicial (1880-1930) circunscribe un periodo de descubrimiento de la actividad. Ante un Estado con extensos territorios despoblados, se comenzó a desarrollar un interés gubernamental por el turismo desde una dimensión territorial, como asegurador de la soberanía nacional. A tal fin, la elite gobernante inició el desarrollo de infraestructura básica, en muchos casos a partir de una alianza con el capital extranjero, que sirvió de sostén a la expansión del turismo en el siguiente periodo. En este entonces la actividad era minoritaria e inequitativa en términos sociales, con un crecimiento limitado e impactos puntuales a nivel territorial. La reducida participación estatal en el área, fue suplida por el destacado accionar de actores privados, que adquirieron una posición preponderante a lo largo del periodo.

La Fase de Expansión (1930-1976) refiere al proceso de democratización de las prácticas turísticas, que permitió un crecimiento exponencial del turismo nacional, a partir de una estructura social ascendente. Concierne a un momento de fuerte intervención estatal, que comenzó a centralizar las diversas acciones públicas llevadas a cabo en el sector desde una dimensión social, a partir de la visibilidad política que adquirieron los colectivos obreros como sujetos de derechos. Los decisores públicos articularon por primera vez capacidades de gobierno específicas, significando la institucionalización del turismo como problema público, a partir de la generalización del derecho a las vacaciones pagadas. El turismo social adquiere una importancia preponderante en el área a parir del peronismo, que impulsa acciones específicas, incluyendo la construcción de complejos turísticos propios.

En este periodo se creó el primer organismo con competencias específicas y se impulsaron las bases normativas del sistema turístico argentino, que establecieron un marco de ordenación en áreas clave del sector. A diferencia de lo que sucede con las primeras políticas turísticas europeas, que desde sus inicios se dirigen principalmente a la promoción en el exterior y a la captación de inversión internacional, la consolidación de la política argentina se da a partir de los flujos turísticos internos. Las grandes distancias con los centros turísticos emisivos, el escaso desarrollo tecnológico en el ámbito de los transportes y las comunicaciones y el 
retrasado proceso de democratización de las prácticas turísticas de los países vecinos, dejan al turismo receptivo en un segundo plano, hasta mediados de 1970.

En la Fase de Reformulación, que transcurre desde 1976 hasta la actualidad, la cuestión turística se adapta a las ideas de desregulación y privatización. Los decisores públicos adoptan un enfoque menos intervencionista en el área, encontrando las referencias necesarias en las recomendaciones de los organismos internacionales. El arribo de las principales cadenas hoteleras y turoperadores constituye el reflejo de un sector turístico ya consolidado, que comienza a exhibir una destacada participación en la política turística nacional a partir de mesas de colaboración y concertación, que institucionaliza el propio Estado. El turismo se constituye en un factor de crecimiento económico e internacionalización, a partir del fortalecimiento del turismo receptivo, que pasa a concentrar una cantidad creciente de los recursos afectados al área, ocupando una posición preponderante entre las políticas sectoriales. El periodo de poscrisis del año 2001, circunscribe un proceso definido dentro de esta última fase, que consolida la función económica asociada al impulso de la actividad a partir de un reposicionamiento del Estado como actor clave en la arena turística. A diferencia de la intervención estatal de mediados del siglo Xx, la Administración cumple una función de facilitación, que promueve la participación de actores públicos y privados, en el diseño, implementación y evaluación de la política turística nacional. En este marco se sanciona la nueva Ley Nacional de Turismo y el área pasa a constituir un órgano autónomo de rango ministerial por primera vez en la historia argentina.

\section{REFERENCIAS BIBLIOGRÁFICAS}

Campodónico, Rossana y de Cunha, Nelly. (2012). Aportes a la conformación de la imagen turística de Uruguay (1930-1955). En Fortalecimiento e Integración de la Investigación en Turismo en América Latina. Trabajo presentado en el V Congreso Latino-Americano de Investigación Turística, San Pablo, Brasil. 
Capanegra, César (2011). La construcción social del turismo como factor de desarrollo, Argentina 1958-2005. En Consolidar el Saber para la Acción en Turismo. Trabajo presentado en las XI Jornadas Internacionales y $\mathrm{V}$ Simposio Internacional de Investigación-Acción en Turismo, Mar del Plata, Argentina.

Cavarozzi, Marcelo (1983). Autoritarismo y democracia: 1955-1983. Buenos Aires: Centro Editor de América Latina.

Ferrer, Aldo (2010). El futuro de nuestro pasado. La economía argentina en su segundo centenario. Buenos Aires: Fondo de Cultura Económica

Franco, Rolando \& Lanzaro, Jorge (Coords.) (2006). Política y Políticas Públicas en los procesos de reforma de América Latina. Buenos Aires: Miño y Dávila.

Gelambí Torrel, Mónica (2006). La terminación de políticas públicas. En Pérez Sánchez, Margarita (Ed.). Análisis de Políticas Públicas, (pp.197-220). Granada: Universidad de Granada.

Jafari, Jafar (2002). Turismo. En Jafari, Jafar. Enciclopedia de turismo, (pp. 635-636). Madrid: Editorial Síntesis

James, Daniel (2010). Resistencia e integración: el peronismo y la clase trabajadora argentina, 1946-1976. Buenos Aires: Siglo Veintiuno Editores.

Mantero, Juan Carlos (1997). Turismo: la opción incluyente. Aportes y Transferencias, 1 (2), 119-136.

Matthews, Harry \& Richter, Linda (1991). Political Science and tourism. Annals of Tourism Research, 18, 120-135.

Oszlak, Oscar \& O’donnell, Guillermo (1995). Estado y políticas estatales en América Latina: hacia una estrategia de investigación. Redes, 4 (2), 99-128.

Pastoriza, Elisa. (2011). La conquista de las vacaciones. Breve historia del turismo en la Argentina. Buenos Aires, Edhasa.

Pérez Sánchez, Margarita (Ed.) (2010). Análisis De Políticas Públicas. Granada: Universidad De Granada.

Rapoport, Mario (2010). Las políticas económicas de la Argentina. Una breve historia. Buenos Aires: Booket

Schenkel, Erica y Almeida, Fernando (2015). La política turística y la intervención del Estado. El caso de Argentina. Perfiles Latinoamericanos. FLACSO, Tlalpan (México). ISSN: 01887653, 46 (23), 197-221. 
Schenkel, Erica (2017). Política turística y turismo social. Una perspectiva latinoamericana Buenos Aires: CLACSO CICCUS.

Schlüter, Regina (2003). El turismo en la Argentina. Del balneario al campo. Buenos Aires: Centro de Investigaciones y Estudios Turísticos.

Scott, Noel (2011). Tourism Policy: A Strategic Review. Oxford: Goodfellow Publishers Limited.

Torrado, Susana. (2010). El costo social del ajuste (Argentina 1976-2002). Buenos Aires: Edhasa.

\section{OTRAS FUENTES}

Memoria detallada del estado de la Nación del año 2003. Jefatura de Gabinete de Ministros. Buenos Aires: JGM.

Ley Nacional N¹8.610/70. Boletín Oficial de la República Argentina. Buenos Aires, 5 de marzo de 1970.

Ley Nacional N¹8.828/70. Boletín Oficial de la República Argentina. Buenos Aires, 19 de noviembre de 1970.

Ley Nacional N¹8.829/70. Boletín Oficial de la República Argentina. Buenos Aires, 19 de noviembre de 1970.

Ley Nacional N¹9.644/72. Boletín Oficial de la República Argentina. Buenos Aires, 2 de junio de 1972.

Ley Nacional No 14.574/58. Boletín Oficial de la República Argentina. Buenos Aires, 11 de noviembre de 1958.

Plan Federal Estratégico de Turismo Sustentable. Actualización 2011. Argentina. PETS. Turismo 2020. Ministerio de Turismo de la Nación, 2011. Buenos Aires.

Plan Federal Estratégico de Turismo Sustentable. Turismo 2016. Secretaría de Turismo de la Nación, 2005. Buenos Aires.

Plan Quinquenal 2. Presidencia de la Nación, 1953. Buenos Aires.

Decreto Nº 1.297/06. Boletín Oficial de la República Argentina. Buenos Aires, 29 de septiembre de 2006.

Decreto N¹.365/09. Boletín Oficial de la República Argentina. Buenos Aires, 2 de octubre de 2009.

Decreto N 1.366/09. Boletín Oficial de la República Argentina. Buenos Aires, 2 de octubre de 2009. 
Decreto $N^{\circ}$ 1.458/09. Boletín Oficial de la República Argentina. Buenos Aires, 13 de octubre de 2009.

Decreto N 919/10. Boletín Oficial de la República Argentina. Buenos Aires, 30 de junio de 2010.

Decreto N921/10. Boletín Oficial de la República Argentina. Buenos Aires, 30 de junio de 2010.

Ley Nacional N²5.997/05. Boletín Oficial de la República Argentina. Buenos Aires, 7 de enero de 2005.

Decreto No 2.025/08. Boletín Oficial de la República Argentina. Buenos Aires, 26 de noviembre de 2008. 